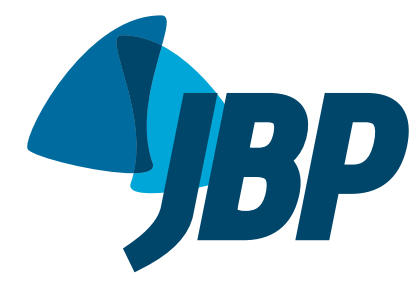

\title{
High-resolution computed tomography findings of pulmonary tuberculosis in lung transplant recipients
}

Irai Luis Giacomelli1, Roberto Schuhmacher Neto', Carlos Schuller Nin'1, Priscilla de Souza Cassano', Marisa Pereira', José da Silva Moreira', Douglas Zaione Nascimento', Bruno Hochhegger ${ }^{1}$

1. Complexo Hospitalar Santa Casa de Porto Alegre, Porto Alegre (RS) Brasil.

Submitted: 5 October 2016. Accepted: 17 March 2017.

Study carried out at the Complexo Hospitalar Santa Casa de Porto Alegre, Porto Alegre (RS) Brasil.

\begin{abstract}
Objective: Respiratory infections constitute a major cause of morbidity and mortality in solid organ transplant recipients. The incidence of pulmonary tuberculosis is high among such patients. On imaging, tuberculosis has various presentations. Greater understanding of those presentations could reduce the impact of the disease by facilitating early diagnosis. Therefore, we attempted to describe the HRCT patterns of pulmonary tuberculosis in lung transplant recipients. Methods: From two hospitals in southern Brazil, we collected the following data on lung transplant recipients who developed pulmonary tuberculosis: gender; age; symptoms; the lung disease that led to transplantation; HRCT pattern; distribution of findings; time from transplantation to pulmonary tuberculosis; and mortality rate. The HRCT findings were classified as miliary nodules; cavitation and centrilobular nodules with a tree-in-bud pattern; ground-glass attenuation with consolidation; mediastinal lymph node enlargement; or pleural effusion. Results: We evaluated 402 lung transplant recipients, 19 of whom developed pulmonary tuberculosis after transplantation. Among those 19 patients, the most common HRCT patterns were ground-glass attenuation with consolidation (in 42\%); cavitation and centrilobular nodules with a tree-in-bud pattern (in 31.5\%); and mediastinal lymph node enlargement (in 15.7\%). Among the patients with cavitation and centrilobular nodules with a tree-in-bud pattern, the distribution was within the upper lobes in $66.6 \%$. No pleural effusion was observed. Despite treatment, one-year mortality was 47.3\%. Conclusions: The predominant HRCT pattern was ground-glass attenuation with consolidation, followed by cavitation and centrilobular nodules with a tree-in-bud pattern. These findings are similar to those reported for immunocompetent patients with pulmonary tuberculosis and considerably different from those reported for AIDS patients with the same disease.
\end{abstract}

Keywords: Lung transplantation; Diagnostic imaging; Mycobacterium infections; Thoracic diseases; Tomography, X-Ray computed/methods; Tuberculosis, pulmonary.

\section{INTRODUCTION}

Lung transplantation has become an established technique for the treatment of end-stage lung disease in adults, and the number of procedures performed each year has grown, as has the number of transplantation centers. ${ }^{(1,2)}$ However, respiratory infection continues to be one of the major concerns in solid organ transplant recipients, clearly constituting a major cause of morbidity and mortality in that population. ${ }^{(2)}$

Tuberculosis is a common infectious disease among humans. In 2014, 9.6 million people worldwide developed tuberculosis and 1.5 million of those people died, $95 \%$ of all tuberculosis-related deaths occurring in low- or middle-income countries. ${ }^{(3)}$ Solid organ transplant patients are more susceptible to tuberculosis infection than are individuals in the general population, the incidence being 20-74 times greater in the former group and the lungs being the most common site of infection. $(4,5)$
Pulmonary tuberculosis is diagnosed on the basis of direct examination (sputum smear microscopy), culture for Mycobacterium tuberculosis, and radiological findings suggestive of the disease. ${ }^{(6)}$ Therefore, the interpretation of imaging findings consistent with tuberculosis is key for early diagnosis and treatment.

Chest CT is one of the main modalities used in cases of clinical suspicion of pulmonary tuberculosis, especially when initial X-rays are normal or when the individual is immunosuppressed, as is the case in AIDS patients and transplant recipients. Some studies have shown that CT is superior to chest X-ray in the initial evaluation of tuberculosis patients. ${ }^{(7,8)}$ Tuberculosis can have a variety of presentations on $\mathrm{CT}^{(9)}$

Some authors have previously studied pulmonary tuberculosis in transplant recipients. ${ }^{(10-13)}$ However, there have been no studies focusing on CT patterns of pulmonary tuberculosis in lung transplant patients. Therefore, the 
present study aimed to determine the presentations of pulmonary tuberculosis seen on HRCT scans of lung transplant recipients.

\section{METHODS}

This was a descriptive study in which we reviewed data related to 402 lung transplant recipients who underwent transplantation at one of two hospitals in southern Brazil between January of 1990 and August of 2015 . This study was approved by the local institutional review board and by the Research Ethics Committee of Plataforma Brasil (Protocol no. 512.215). The inclusion criteria were testing positive for $M$. tuberculosis in sputum culture, testing positive for $M$. tuberculosis in culture from BAL fluid or a lung biopsy sample, and having had an HRCT scan performed after diagnosis. Based on a review of clinical and laboratory data, we excluded patients diagnosed with mycosis or concomitant viral infections potentially affecting the lungs, including cytomegalovirus infections. We collected data regarding the following: gender; age; symptoms; the lung disease that led to transplantation; HRCT lung pattern; HRCT lung distribution pattern; time from transplantation to pulmonary tuberculosis; and mortality rate.

All HRCT scans were acquired in a 64-slice multidetector scanner (LightSpeed VCT; GE Healthcare, Waukesha, WI, USA), with the following parameters: tube voltage, $120 \mathrm{kVp}$; tube current, $250 \mathrm{mAs}$; rotation time, $0.8 \mathrm{~s}$; and pitch, 1.375 . The technical parameters included inspiratory volumetric acquisition with $1 \mathrm{~mm}$ collimation in 1-mm increments using a high-spatialfrequency reconstruction algorithm. Images were obtained with mediastinal window settings (width, 350 to $450 \mathrm{HU}$; level, 20 to $40 \mathrm{HU}$ ) and parenchymal window settings (width, 1200 to $1600 \mathrm{HU}$; level, -500 to $-700 \mathrm{HU})$, and reconstructions were performed in the axial and coronal planes.

Two chest radiologists, with more than 10 years of experience and both blinded to the clinical status of the patients, independently assessed the HRCT scans in random order. After the two radiologists had conducted their independent analyses, they reviewed the images together with a third chest radiologist (with $>30$ years of experience) in order to reach a final consensus decision. For each patient, reviewers identified one predominant CT pattern, according to the criteria set forth in the Fleischner Society's Glossary of Terms. ${ }^{(14)}$ The HRCT findings were categorized as follows: miliary nodules; cavitation and centrilobular nodules with a tree-in-bud pattern; ground-glass attenuation with consolidation; mediastinal lymph node enlargement; or pleural effusion.

A nodule was defined as a rounded or irregular, ill- or well-defined opacity with a diameter $\leq 3 \mathrm{~cm}$. ${ }^{(14)}$ Mediastinal and hilar lymph nodes varied in size from sub-CT resolution to $10 \mathrm{~mm}$. Mediastinal lymph node enlargement was defined as mediastinal lymph nodes $>10 \mathrm{~mm}$ in diameter on their short-axis, as demonstrated by Cascade et al. ${ }^{(15)}$ Cavities were defined as gas-filled spaces, presenting as lucencies or areas of low-attenuation within pulmonary consolidations, masses, or nodules. The tree-in-bud pattern refers to centrilobular branching structures that resemble a budding tree. Ground-glass opacities were defined as hazy areas of increased attenuation, with no obscuration of the underlying vessels. ${ }^{(14)}$ Consolidation was defined as homogeneous opacification of the parenchyma with obscuration of the underlying vessels. The distribution of abnormalities was categorized as focal (when unilobar) or diffuse (when involving more than one lobe), and the findings were stratified by zone (upper, middle, and lower lung). ${ }^{(14)}$ Continuous variables were expressed as mean and standard deviation, whereas categorical variables were expressed as absolute and relative frequencies.

\section{RESULTS}

Among the 402 lung transplant recipients evaluated, we identified 20 who were diagnosed with pulmonary tuberculosis. Of those 20 patients, one was excluded due to coinfection with cytomegalovirus. Therefore, the final sample comprised 19 patients (12 males and 7 females), ranging from 11 to 65 years of age (mean, $33 \pm 18$ years). The underlying diseases that led to transplantation were the following: pulmonary emphysema, in 7 patients (36\%); pulmonary fibrosis, in $7(36 \%)$; silicosis, in $3(15.7 \%)$; and pulmonary hypertension, in $2(10.5 \%)$. All of the patients had asthenia and cough. The mean time from lung transplantation to the diagnosis of pulmonary tuberculosis was $3.2 \pm 1.7$ months.

Table 1 shows the study sample by HRCT pattern, together with the distribution of the findings within the lung. The main HRCT patterns were ground-glass attenuation with consolidation (in $42 \%$ of the patients); cavitation and centrilobular nodules with a tree-in-bud pattern (in 31.5\%); and mediastinal lymph node enlargement (in 15.7\%). The first two are depicted in Figures 1 and 2, respectively. In $66.6 \%$ of patients with cavitation and centrilobular nodules with a treein-bud pattern, the distribution was within the upper lobes. No pleural effusion was observed. Two patients died. In those two patients, the HRCT findings were ground-glass attenuation with consolidation and miliary nodules, respectively.

\section{DISCUSSION}

To our knowledge, this is the first study to describe the HRCT findings of pulmonary tuberculosis exclusively among lung transplant recipients. The presentation of pulmonary tuberculosis was stratified into four patterns: ground-glass attenuation with consolidation; cavitation and centrilobular nodules with a tree-in-bud pattern; mediastinal lymph node enlargement; and miliary nodules. In over $70 \%$ of the cases evaluated, we observed cavitation and centrilobular nodules with 
Table 1. HRCT pattern and distribution of findings by lung zone.

\begin{tabular}{lcc}
\multicolumn{1}{c}{ HRCT pattern } & Prevalence* & Lung zone \\
Ground-glass attenuation with consolidation & $8(42)$ & Upper lobes (in 50.0\%) \\
$\begin{array}{l}\text { Cavitation and centrilobular nodules with a tree-in-bud } \\
\text { pattern }\end{array}$ & $6(31.5)$ & Upper lobes (in 66.6\%) \\
Mediastinal lymph node enlargement & $3(15.7)$ & N/A \\
Miliary nodules & $2(10.5)$ & Random \\
All & $19(100)$ & N/A \\
\hline
\end{tabular}

*Data are expressed as $\mathrm{n}(\%)$.
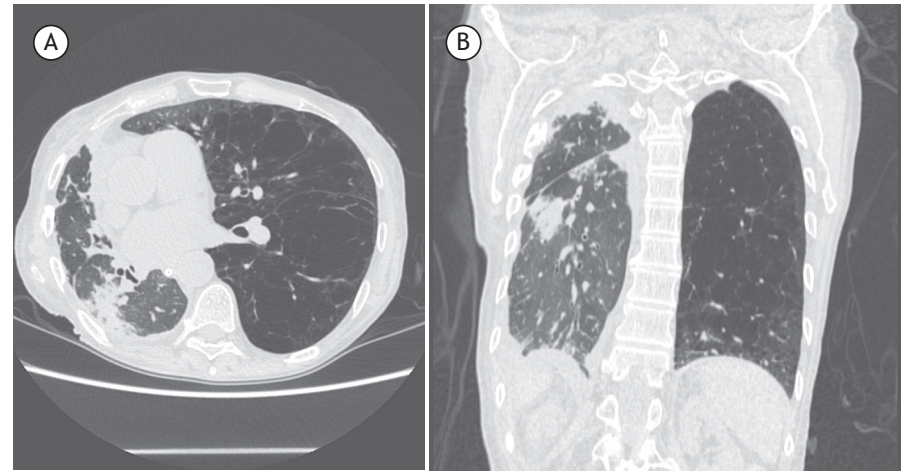

Figure 1. Pulmonary tuberculosis in a 48-year-old man who underwent right lung transplantation. Axial and coronal HRCT scans ( $A$ and $B$, respectively), showing diffuse pleural thickening, peripheral linear opacities, and consolidation in the superior segment of the right lower lobe of the right lung. Note the increased volume of the left lung, due to extensive panacinar emphysema.
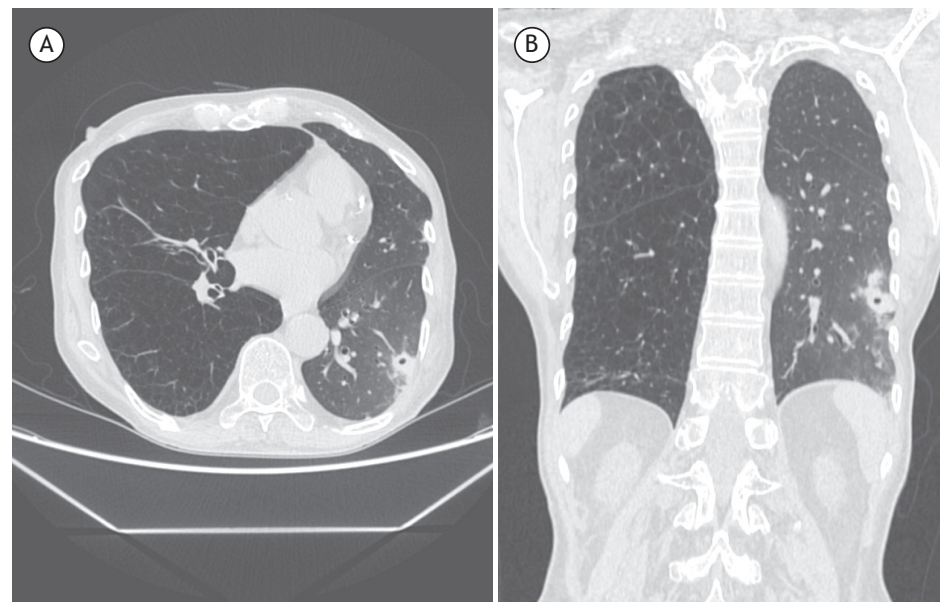

Figure 2. Pulmonary tuberculosis in a 54-year-old man who underwent left lung transplantation. Axial and coronal HRCT scans (A and B, respectively), showing a small, irregular thick-walled cavity in the lateral-basal segment of the left lower lobe and adjacent satellite nodules. Extensive panacinar emphysema can be seen in the right lung.

a tree-in-bud pattern or ground-glass attenuation with consolidation.

Meta-analyses have shown that the average time from solid organ transplantation to tuberculosis infection is 3.5 months, an interval quite similar to that found in our study. ${ }^{(4)}$ All of the patients in our sample were receiving immunosuppression therapy for lung transplantation. The same treatment protocol was followed in every case. The time from lung transplantation to the development of tuberculosis was comparable among the participants, indicating that the sample was fairly homogeneous in terms of the immunosuppressive state of the patients.
None of the patients in our sample showed pleural effusion as a manifestation of pulmonary tuberculosis. In a study that evaluated the radiographic presentations of pulmonary tuberculosis in 226 solid organ transplant recipients, the authors observed pleural effusion in $13 \%$. ${ }^{(4)}$ That discrepancy could be explained by the fact that we evaluated lung transplant recipients exclusively and by local postoperative changes.

In the general population, the most common HRCT patterns of pulmonary tuberculosis are mediastinal lymphadenopathy, cavitation, centrilobular nodules with a tree-in-bud pattern, consolidation, and groundglass opacities, all of which occur predominantly in the 
upper lobes. ${ }^{(16,17)}$ In the present study, we observed the same patterns and also found a predominance of upper lobe findings.

In the general population, the presence of cavities in imaging studies is an important sign of active disease. In a study involving 41 solid organ transplant recipients, Im et al. (18) found that the prevalence of cavities on CT scans was $58 \%$, higher than the $31.5 \%$ observed in our study. Those authors showed that, among individuals with active tuberculosis in the general population, the most common CT finding (in $82-100 \%$ of cases) was centrilobular nodules with segmental distribution, which represents bronchogenic dissemination of the disease. In our study sample, centrilobular nodules occurred in nearly one third of the patients evaluated, the lower incidence potentially being related to the use of immunosuppression therapy.

In comparison with the HIV-negative population, individuals with AIDS are more likely to demonstrate lymph node involvement and miliary disease. ${ }^{(19)}$ Hilar and mediastinal lymph node enlargement occurs in over $60 \%$ of AIDS patients with tuberculosis. $(20,21)$
In our sample, the miliary nodular pattern was observed in only 2 patients (10.5\%), and the mediastinal lymph node enlargement pattern was observed in only $3(15.7 \%)$ These findings suggest that lung transplant recipients with pulmonary tuberculosis present with CT findings that are more similar to those reported for immunocompetent individuals with pulmonary tuberculosis than to those reported for AIDS patients with pulmonary tuberculosis.

One limitation of our study was the small size of the sample. However, this was the largest study to date of pulmonary tuberculosis in lung transplant recipients. In addition, microbiological confirmation was obtained in all of the cases.

In lung transplant recipients with pulmonary tuberculosis, HRCT most often revealed ground-glass attenuation with consolidation or cavitation and centrilobular nodules with a tree-in-bud pattern. The distribution and predominant imaging findings in this patient population are similar to those reported for immunocompetent patients with pulmonary tuberculosis and considerably different from those reported for AIDS patients with pulmonary tuberculosis.

\section{REFERENCES}

1. Toronto Lung Transplant Group. Unilateral lung transplantation for pulmonary fibrosis. N Engl J Med. 1986;314(18):1140-5. https://doi. org/10.1056/NEJM198605013141802

2. Hadjiliadis D, Chaparro C, Gutierrez C, Steele MP, Singer LG Davis RD, et al. Impact of lung transplant operation on bronchiolitis obliterans syndrome in patients with chronic obstructive pulmonary disease. Am J Transplant. 2006;6(1):183-9. https://doi.org/10.1111/ j.1600-6143.2005.01159.x

3. World Health Organization [homepage on the Internet]. Geneva: World Health Organization [updated 2017 Mar; cited 2016 Oct 1]. Media centre. Tuberculosis. [about 7 screens]. Available from: http:// www.who.int/mediacentre/factsheets/fs104/en/

4. Singh N, Paterson DL. Mycobacterium tuberculosis infection in solidorgan transplant recipients: impact and implications for management. Clin Infect Dis. 1998;27(5):1266-77. https://doi.org/10.1086/514993

5. Subramanian A, Dorman S; AST Infectious Diseases Community of Practice. Mycobacterium tuberculosis in solid organ transplant recipients. Am J Transplant. 2009;9 Suppl 4:S57-62. https://doi. org/10.1111/j.1600-6143.2009.02894.x

6. Brasil. Ministério da Saúde. Manual de normas para o controle da tuberculose. 4th ed. Brasilia: o Ministério; 1995

7. Lee KS, Im JG. CT in adults with tuberculosis of the chest: characteristic findings and role in management. AJR Am J Roentegenol 1995;164(6):1361-7. https://doi.org/10.2214/ ajr.164.6.7754873

8. Hatipoğlu ON, Osma E, Manisali M, Uçan ES, Balci P, Akkoçlu A et al. High resolution computed tomographic findings in pulmonary tuberculosis. Thorax. 1996;51(4):397-402. https://doi.org/10.1136/ thx.51.4.397

9. Torre-Cisneros J, Doblas A, Aguado JM, San Juan R, Blanes M, Montejo $\mathrm{M}$, et al. Tuberculosis after solid-organ transplant: incidence, risk factors, and clinical characteristics in the RESITRA (Spanish Network of Infection in Transplantation) cohort. Clin Infect Dis. 2009:48(12):1657-65.

10. Krishnam MS, Suh RD, Tomasian A, Goldin JG, Lai C, Brown K, et al. Postoperative complications of lung transplantation: radiologic findings along a time continuum. Radiographics. 2007;27(4): 957-74. https://doi.org/10.1148/rg.274065141

11. Beigelman C, Sellami D, Brauner M. CT of parenchymal and bronchial tuberculosis. Eur Radiol. 2000;10(5):699-709. https://doi.org/10.1007/

\section{s003300050989}

12. Hemmert $C$, Ohana M, Jeung MY, Labani A, Dhar A, Kessler R, et al. Imaging of lung transplant complications. Diagn Interv Imaging 2014;95(4):399-409. https://doi.org/10.1016/j.diii.2013.09.005

13. Jokerst $\mathrm{C}$, Sirajuddin A, Mohammed TH. Imaging the complications of lung transplantation. Radiol Clin North Am. 2016;54(2):355-73. https://doi.org/10.1016/j.rcl.2015.09.014

14. Hansell DM, Bankier AA MacMahon $H$, McLoud TC, Mülle $\mathrm{NL}$, Remy J. Fleischner Society: glossary of terms for thoracic imaging. Radiology. 2008;246(3):697-722. https://doi.org/10.1148/ radiol.2462070712

15. Cascade PN, Gross BH, Kazerooni EA, Quint LE, Francis IR Strawderman $M$, et al. Variability in the detection of enlarged mediastinal lymph nodes in staging lung cancer: a comparison of contrast-enhanced and unenhanced CT. AJR Am J Roentgenol. 1998;170(4):927-31. https://doi.org/10.2214/ajr.170.4.9530036

16. Yeh JJ, Chen SC, Chen CR, Yeh TC, Lin HK, Hong JB, et al. A high-resolution computed tomography-based scoring system to differentiate the most infectious active pulmonary tuberculosis from community-acquired pneumonia in elderly and non-elderly patients. Eur Radiol. 2014;24(10):2372-84. https://doi.org/10.1007/s00330014-3279-6

17. Andreu J, Cáceres J, Pallisa E, Martinez-Rodriguez M. Radiological manifestations of pulmonary tuberculosis. Eur $J$ Radiol. 2004;51(2):139-49. https://doi.org/10.1016/j.ejrad.2004.03.009

18. Im JG, Itoh H, Shim YS, Lee JH, Ahn JA, Han MC, et al. Pulmonary tuberculosis: CT findings-early active disease and sequential change with antituberculous therapy. Radiology. 1993;186(3):653-60. https:// doi.org/10.1148/radiology.186.3.8430169

19. Saurborn DP, Fishman JE, Boiselle PM. The imaging spectrum of pulmonary tuberculosis in AIDS. J Thorac Imaging. 2002;17(1):28-33. https://doi.org/10.1097/00005382-200201000-00003

20. Castañer E, Gallardo X, Mata JM, Esteba L. Radiologic approach to the diagnosis of infectious pulmonary diseases in patients infected with the human immunodeficiency virus. Eur J Radiol. 2004;51(2):114-29. https://doi.org/10.1016/j.ejrad.2004.03.008

21. Almeida LA, Barba MF, Moreira FA, Rombarda S, Felice SA, Calore EE. Computed tomography findings of pulmonary tuberculosis in adult AIDS patients. Radiol Bras. 2011;44(1):13-9. https://doi. org/10.1590/S0100-39842011000100007 